\title{
ResearchOnline@JCU
}

This is the author-created version of the following work:

Wolstenholme, Jackie, Nozawa, Yoko, Byrne, Maria, and Burke, William (2018) Timing of mass spawning in corals: potential influence of the coincidence of lunar factors and associated changes in atmospheric pressure from northern and southern hemisphere case studies. Invertebrate Reproduction and Development, 62 (2) pp. 98-108.

Access to this file is available from:

https://researchonline.jcu.edu.au/52181/

Please refer to the original source for the final version of this work: 


\section{Timing of mass spawning in corals: potential influence of the coincidence of lunar factors and associated changes in atmospheric pressure from northern and southern hemisphere case studies}

Submitted to: Invertebrate Reproduction \& Development

Jacqueline Wolstenholme ${ }^{\mathrm{a}}$, Yoko Nozawa ${ }^{\mathrm{b}}$, Maria Byrne ${ }^{\mathrm{c}}$ and William Burke $^{\mathrm{d}}$

a Library and Information Services, James Cook University, Townsville, Australia; ORCID: 0000-0002-2414-3298; Twitter: @ajacquelineae

b Biodiversity Research Center, Academia Sinica, Taipei, Taiwan; ORCID: 0000-00016124-6045

c School of Medical Sciences and School of Life and Environmental Sciences, University of Sydney, Sydney, Australia; ORCID: 0000-0002-8902-9808

d Discipline of Physiology, University of Sydney, Sydney, Australia

Corresponding author: Jacqueline Wolstenholme jackie.wolstenholme@jcu.edu.au

Keywords: gamete release; lunar rhythms; coral reefs, Japan, Great Barrier Reef, 


\title{
Timing of mass spawning in corals: potential influence of the coincidence of lunar factors and associated changes in atmospheric pressure from northern and southern hemisphere case studies
}

\begin{abstract}
Synchronized multispecies mass spawning events are striking features of reproduction in corals. This synchronous gamete release of thousands of animals over vast stretches of reef is thought to be cued by rhythms of the Moon. However, the mechanisms are not fully understood. We propose an explanation that may contribute to understanding this mechanism, that spawning is triggered by the coincidence of two factors, each in different lunar rhythms. We investigate this proposal in case studies using seven years of coral spawning data from two locations: Kochi, Japan and Lizard Island, Australia. Our calculations show that a feature in a lunar synodic rhythm (the third quarter) will synchronise with a feature in a lunar non-synodic rhythm (the zero declination) usually once, although occasionally twice in a year. Supported by data on the date of spawning from the two locations, we suggest that this coincidence of lunar factors exerts an important influence on the timing of annual mass spawning in corals. This coincidence may be associated with low atmospheric pressure. Spawning at the time of the third lunar quarter may favour fertilisation success due to the reduced currents during neap tides associated with the lower gravitational pressure of the lunar quarters.
\end{abstract}

\section{Impact Statement}

- Coral mass spawning is an annual natural phenomenon in which reef corals synchronise their reproduction over large geographic areas.

- These events are both spectacular and intriguing: spectacular because they resemble underwater snowstorms of eggs and sperm, and intriguing because so many tiny polyps co-ordinate their reproduction within a period of just a couple of days.

- Understanding the timing of these events has important commercial implications for the diving tourism industry.

- Understanding the timing of these events also has important environmental implications for improving the management of reefs e.g. restricting shipping to avoid disruption of the eggs and sperm which concentrate at the water surface in the days after spawning events.

- Our research proposes a novel model which may assist to more accurately predict the nights which corals spawn. 
- We suggest that the coincidence of two factors, the timing of the third quarter moon with the timing of another previously unconsidered factor i.e. the passing of the moon over the equator, could influence when corals mass spawn.

- Our preliminary evidence suggests that this coincidence may be associated with low atmospheric pressure and may be a trigger for corals to spawn.

- To explain this proposal, we use data from two locations: Kochi, Japan, and Lizard Island, Great Barrier Reef, Australia.

\section{Introduction}

Lunar cycle-related rhythms of reproduction in marine invertebrates, with annual spawning coinciding with a particular moon phase are a long-recognised phenomena that have generated a wealth of hypotheses as to the controlling factors (Friedlaender 1898; Okuda 1939; Korringa 1947; Dan and Kubota 1960; Keith et al. 2016). As these annual spawning events are also associated with other factors such as seasonal/day length (solar) cycles, insolation, tides, low wind speeds and increasing sea surface temperature, some of which co-vary with lunar cues, it remains a challenge to understand the influence of celestial forces on reproduction in the sea (Mercier and Hamel 2010, 2014; van Woesik 2010; Sweeney et al. 2011; Keith et al. 2016).

Synchronized multispecies spawning events are striking features of benthic marine invertebrate communities from the tropics to boreal habitats (Babcock et al. 1986, 1992, 1994; Himmelman et al. 2008; Sweeney et al. 2011). Although reproduction of marine invertebrates occurs outside of mass spawning events, predictable synchronous spawning has been widely documented in corals and many other species of marine invertebrates, including polychaetes (e.g. Eunice viridis: Korringa 1957; Caspers 1984), holothurians (Babcock et al. 1992; Balogh et al. 2018) and soft corals (Alino and Coll 1989). Among the best studied are the spectacular annual coral mass-spawning events when 100 or more scleractinian corals release gametes during one or a few nights in the week after the full moon (Harrison et al. 1984; Babcock et al. 1986, 1992, 1994; Baird et al. 2009). 
The month of annual coral mass spawning events in both the northern and southern hemispheres can be reliably predicted (Levitan et al. 2004; Wolstenholme 2004; Baird et al. 2009; Nozawa 2012). Harrison et al. (1984) provided the first documented evidence of synchronous multispecies spawning in corals with the event occurring in late spring on the Great Barrier Reef (GBR), Australia. In other locations (e.g. Okinawa, Caribbean) many species of scleractinian corals also mass-spawn in late spring at predictable times with respect to the lunar cycle (e.g. Hayashibara et al. 1993; Levitan et al. 2004; Nozawa, 2012).

Coral mass-spawning events can usually be predicted with an accuracy window of a few days within the months of spring or early summer. For instance, Harrison et al. (1984) concluded that spawning of corals on the GBR occurs four or five nights after one or two full moons in spring.

The time of day of gamete release in mass spawning corals is also predictable. The proximate trigger for the fine-tuning of the hour of spawning is suggested to be luminance near or after sunset, and characteristic for each species. For example, Acropora tenuis spawns on dusk (Harrison et al. 1984) and A. monticulosa was found to spawn 5 - 6.25 hours after sunset (Wolstenholme 2004), while the majority of species spawn between these times (e.g. Harrison et al. 1984; Babcock et al. 1986). It is suggested that decreasing light in deeper water causes deeper corals to spawn before those living in shallower water (Levitan et al. 2004). Thus, the final discrete trigger for gamete release may be related to crepuscular or optical phenomena around sunset (Levitan et al. 2004; Sweeney et al. 2011) or moonlight (Kaniewska et al. 2015).

Therefore, the timing of mass spawning events in corals can be seen as occurring in three time frames: time of year, time of month and time of day. For time of year, the spawning season is the result of gametogenesis and gamete maturation and 
appears to be largely entrained by temperature (Guest et al. 2005; Keith et al. 2016). For the time of month, there is the mechanism whereby corals spawn in a single month, or in the case of split spawning years, in consecutive months (Willis et al. 1985; Baird et al. 2009). Finally, there are mechanisms that limit spawning to a particular time of day. The present study is concerned with the second time frame, specifically with why massspawning of multiple species is limited to a few consecutive days in certain months of the year and how the rhythms of the Moon may be a potential influence.

Atmospheric pressure is another element that has not previously been considered as a possible influence of the timing of coral mass spawning. Li et al. (2011) report a relationship between lunar gravitational influence on the Earth’s rotation and atmospheric pressure fields. They found that lunar gravitational forces were more than twice that of solar gravitational forces. Li et al. (2011) also found that lunar declination was the most important factor in determining tidal cycles in atmospheric pressure.

Here we present the application of a previously unconsidered perspective on elements which may contribute to our understanding of the days when corals mass spawn. We refer to this new perspective as the 'Coincidence of Factors' model. This model was first proposed by Burke et al. (2013) in a study of the relationship between visual acuity in humans and the lunar synodic rhythm. Here, we extend the model, using corals as a marine invertebrate case study to investigate the synchronous spawning of thousands of individuals within the brief temporal window of a few days. The Coincidence of Factors model assumes that spawning is triggered by the coincidence of two factors, each in a different lunar rhythm, one factor with a synodic rhythm and the other factor with a non-synodic rhythm (see methods). We compare and analyse the coincidence of the two lunar rhythms in two case studies using previously published data on spawning dates from Kochi, Japan and Lizard Island, Australia (Wolstenholme 
2004; Nozawa 2012). We investigate the influence of atmospheric pressure on the timing of coral spawning because of the close association between zero declinations and atmospheric pressure ( $\mathrm{Li}$ et al. 2011). We also examined wind speed because calm to moderate wind speeds are reported to influence the timing of coral mass spawning events (van Woesik 2010; Keith et al. 2016).

\section{Materials and Methods}

\section{Lunar rhythms}

There are six lunar rhythms, all of which depend on the movement of the Moon through space. The best-known rhythm is the synodic rhythm, consisting of the new moon, first quarter, full moon and third quarter, with a period of 29.53 days (Espenak 2012). The synodic rhythm includes variations in light and gravity which act on the Earth and biological rhythms of many marine species (Tessmar-Raible et al. 2011).

We make use of this synodic rhythm as the first potential factor influencing spawning in our Coincidence of Factors model because this rhythm has frequently been proposed to influence the timing of reproduction in corals (reviewed in Harrison et al. 1984, 2011; Baird et al. 2009). Although the day when mass spawning occurs in corals has commonly been referenced with respect to the full moon, we use the third quarter as reference because we find that spawning frequently occurs exactly on or close to those days.

It is probable that all non-synodic rhythms also have a gravitational effect on the Earth (Burke et al. 2013). Of these rhythms, as the second factor in our model, we use the lunar declination rhythm which has a period of 27.32 days (Espenak 2012). We use the lunar declination rhythm because in a previous paper (Burke et al. 2013), it was reported that this rhythm acted as the second factor in influencing changes in human 
visual acuity on an annual cycle.

\section{Time of spawning at Kochi and Lizard Island}

We used published dates of spawning for corals from the Northern Hemisphere in Kochi, Japan (Nozawa 2012) and the Southern Hemisphere from Lizard Island, Great Barrier Reef, Australia (Wolstenholme 2004) as case studies to investigate our model. The four years of spawning data from Kochi (2006-2009) and three years of spawning data from Lizard Island (1999-2001) provided the opportunity to compare the relationship of spawning with the lunar cycle in the two locations.

The spawning data collected from the Kochi and Lizard Island studies were originally collected for differing purposes: in Kochi to examine the influence of temperature and other environmental factors on coral reproduction (Nozawa 2012), and at Lizard Island to examine the potential for colonies of the same and different species to interbreed (Wolstenholme 2004). Importantly, both studies provide detailed evidence of the timing (date) of mass spawning in relation to the lunar cycle for each location. For Kochi we used the data for the large spawning events (see Nozawa 2012) as we focussed on large mass spawning. All the data in Wolstenholme (2004) are from large spawning events at Lizard Island. In addition to the data for the mass spawning period at Lizard Island, Wolstenholme (2004) also provided dates on the timing of coral spawning in the subsequent summer months after the mass spawning period, enabling additional data for investigating the Coincidence of Factors model.

The spawning data for Kochi were collected from 2006-2009 in July and August for 139 colonies as described by Nozawa (2012). The dates of spawning for each spawning colony are listed in Table S1 (http://doi.org/10.4225/28/59658419a8ee9). Data were recorded as repeated, monthly field observations of the same 40 tagged colonies: 10 colonies of each of four species, with two colonies of one species dying in 
2008 and a third colony dying in 2009. Colonies containing mature eggs just before the predicted spawning season in July 2006 were monitored by chiselling off small fragments. In 2006 and 2007, gametes were also collected in “egg-capture devices”. In 2008 and 2009, data were only collected by observation on night scuba dives around the third lunar quarter. From October 2006 through to April 2007, ten colonies of each of the four species were also checked every second month to confirm that colonies did not contain mature gametes during months when spawning was not observed.

The spawning data for Lizard Island were collected from 1999-2001 in November to February. Data were recorded for 71 colonies which spawned in laboratory aquaria (Wolstenholme 2004). The colonies were collected and placed in the aquaria between one to five days after the full moon. The 71 colonies, for which spawning data were recorded, were $89 \%$ of the monitored colonies (Table 5, Wolstenholme 2004). The dates of spawning for each spawning colony are listed in Table S2 (http://doi.org/10.4225/28/59658419a8ee9). Complementary surveys confirmed that the spawning in the aquaria corresponded with the timing of gamete maturity in the field (Figure 3, Wolstenholme 2004). The peak of spawning at Lizard Island was consistently 6-8 nights after the full moon, with there being no evidence of mass spawning occurring prior to these dates e.g. colonies in the field with mature gametes were abundant, and there was no coral spawn on beaches. These observations were in association with the fertilisation study by Wolstenholme (2004). Parallel observations from divers on reefs at Lizard Island corresponded with the major nights of mass spawning, with colonies in the aquaria spawning a couple of hours after field observations (data not shown). 


\section{Lunar data}

The synodic rhythm is based on the time taken for the Moon to orbit the Earth, and return to the same phase e.g. the full moon (see video in the Supplemental Data: http://doi.org/10.4225/28/59658419a8ee9). We applied the synodic rhythm by comparing the timing of the third quarter moon phases to the timing of spawning.

The lunar declination is a sidereal rhythm based on the angle that the Moon makes with the Earth's equator. We use the dates of the zero declinations, i.e. when the Moon passes over the equator as it moves from the northern to the southern hemisphere and the southern to the northern hemisphere.

The dates of the third lunar quarter moon phases were obtained from the United States Naval Observatory (USNO) Astronomical Applications Department portal (http://aa.usno.navy.mil/data/docs/MoonPhase.php). The dates of the zero declinations were obtained from the web-based interface of the NASA Jet Propulsion Laboratory (JPL) California Institute of Technology HORIZONS system (https://ssd.jpl.nasa.gov/horizons.cgi) with assistance from HackyHour @ James Cook University (2017). The following settings were used to define the datasets: Ephemeris Type: Observer; Target Body: Moon [Luna] [301]; Table Settings: Quantities=1, CSV format=Yes, Display/Output: download/save (plain text file). The Observer Location was user defined as Kochi: 132deg43’57.0”E, 32deg46’44.0”N and Lizard Island: 145deg27’37.4”E, 14deg40’05.5”S. In the downloaded data, declination values are positive for the northern hemisphere and negative for the southern hemisphere. The zero declination dates are the dates when the values change between positive and negative values. 


\section{Atmospheric pressure}

Atmospheric pressure and wind speed data were obtained for the two locations of this study. For the atmospheric pressure data, the year range of 2000-2016 was used because this includes the years of the Kochi and Lizard Island coral spawning data up to the most recent complete year. Data for Shimizu, Kochi were obtained from the Japan Meteorological Agency (http://www.jma.go.jp/jma/indexe.html) using the 'grouped by mean daily average’ option. Data for Lizard Island were obtained from the Australian Institute of Marine Science Historic Data Tool (http://data.aims.gov.au/aimsrtds/datatool.xhtml) using the 'grouped by mean daily average with rule-based quality control applied’ option. Atmospheric pressure data for Lizard Island were only available from 2010 and so atmospheric pressure data from nearby Agincourt Reef (156km south of Lizard Island) was used as a proxy. Agincourt Reef is the closest location for which the meteorological data were available. A large proportion of the Agincourt Reef data contained erroneous or missing values for 1999, the first year of the Lizard Island coral spawning data and so this year was not included in the analysis. The wind speed data were obtained from the same sites using the same settings as for the atmospheric pressure data, but only for the years of the spawning data (2006-2009 for Kochi and 1999-2001 for Agincourt Reef).

\section{'Coincidence of Factors' model}

To investigate the Coincidence of Factors model, we examined whether the coincidence, or near coincidence, of the third lunar quarter synodic rhythm and zero declination sidereal rhythm could influence the timing of when corals mass spawn. We use these two rhythms because Burke et al. (2013) found that the combination of the lunar quarter and zero declination are associated with a rare physiological event i.e. a change in human visual acuity, occurring about once per year (see Burke et al. 2013). 
The synodic rhythm is the factor most often associated with spawning in corals, usually expressed as a number of days that spawning occurred after the full moon e.g. 39 days (Table 5, Wolstenholme 2004). The synodic rhythm has a period of 29.53 days. This is the number of days between e.g. successive full moons or third lunar quarters.

The second factor we examined is the zero declination. This rhythm has a period of 27.32 days. The lunar declination describes the path of the Moon as it moves from a latitude of about $28^{\circ} \mathrm{N}$ to about $28^{\circ} \mathrm{S}$ and back again to $28^{\circ} \mathrm{N}$. Thus, in the course of the 27.32 days, the Moon crosses the Earth’s equator twice going from north to south, symbolized as $\mathrm{E}(\mathrm{N}>\mathrm{S})$, and from south to north, $\mathrm{E}(\mathrm{S}>\mathrm{N})$.

We use the expression 'Coincidence of Factors' although the synchrony may not be an exact coincidence, just closeness in time. Figure 1 and the accompanying video (Supplemental Data: http://doi.org/10.4225/28/59658419a8ee9) explain how the coincidence comes about. In this figure, the large circles represent equatorial crosssections of the Earth. The small black circles are symbols for the third lunar quarter (LQ) with a period 29.53 days. The small black rectangles are symbols for the lunar zero declinations (non-synodic: ZD: period 27.32 days). The symbols orbit the Earth in a clockwise direction. In Figure 1 (A) the symbols are shown in a simultaneous position at the base of the figure as a starting point for simplicity. After one lunar cycle (Figure 1 (B)) the LQ symbol is back in its original position (29.53 days) but the ZD symbol is 2.21 days ahead (29.53 - 27.32). After 3 lunar cycles (Figure 1 (C)) the ZD symbol is now 6.63 (3x2.21) days ahead. Figure 1 (D) and Figure 1 (E) show the ZD symbols after 6 and 9 lunar cycles. At 13 lunar cycles (Figure 1 (F)) the ZD symbol is now 28.73 (2.21 x 13) days ahead. It is now very close to the LQ symbol $(29.53-28.73=0.80$ day), and we now ask if this interval might be close enough to signal a significant correlation with spawning. After 14 lunar cycles the ZD symbol is now 30.94 (2.21 x 
14) days ahead (open rectangle in Figure $1(\mathrm{~F})$ ), but this is equivalent to being only 1.41

(30.94 - 29.53) days away from the LQ symbol and therefore again we query if this interval could correlate with spawning. It is important to note that the presentation of the model in Figure 1 and the video (Supplemental Data:

http://doi.org/10.4225/28/59658419a8ee9) are based on the Lizard Island and the southern hemisphere geography. A slightly different diagram for the northern hemisphere would be similar in showing closeness of the LQ and ZD at 13 and 14 lunar cycles.

\section{Results}

\section{Timing of mass spawning}

At Kochi, major coral spawning (see Nozawa 2012) occurred within a range of seven days, from three days before and four days after the third lunar quarter with $68.6 \%$ of colonies spawning on or within one day of the third lunar quarter (Figure 2A).

Similarly, at Lizard Island, spawning also occurred around the third lunar quarter, ranging from four days before and one day after this phase with $80.8 \%$ of colonies spawning on or within one day of the third lunar quarter (Figure 2B). This timing at Lizard Island around the third lunar quarter included the mass spawning events as well as the spawning events recorded in the subsequent summer months.

\section{'Coincidence of Factors' model}

Figure 3 shows the temporal differences between the third quarters and the zero declinations as the Moon moves around the Earth during successive cycles in the course of a year. These cycles are similar to the placements of the Moon as shown in Figure 1 but they show the exact data taken from the records in both locations: Kochi and Lizard Island. Note that spawning occurs in the summer, the months which are in the centre of 
the graphs for Kochi (Figure 3A and Figure 3B: July, August, see Table S1:

http://doi.org/10.4225/28/59658419a8ee9), and the start and end of the calendar year for Lizard Island (Figure 3C and Figure 3D: November, December, January, February, see Table S2: http://doi.org/10.4225/28/59658419a8ee9). Superimposed on Figure 3A and Figure $3 \mathrm{C}$ are the spawning events at the times of their occurrence. The spawning times are indicated by the vertical dashed lines and the LQ - ZD reference is indicated by the point where these lines intersect the plotted curves. In agreement with the predictions of our model, it can be seen that for both locations, the spawning events occur where the lunar quarter- zero declination interval (LQ - ZD) is close to zero. The time between the lunar quarters and zero declinations is similar to those calculated in Figure 1, with the longest time being 4 days (Table 1).

\section{Zero declination}

Comparison of the zero declination with other latitudes for the Moon's position i.e. in the extreme northern and southern latitudes $\left(\sim 28^{\circ}\right)$ and both zero declinations $(\mathrm{E}(\mathrm{N}>\mathrm{S})$ and $\mathrm{E}(\mathrm{S}>\mathrm{N})$ indicates the close association between the zero declination and timing of spawning (Figure 3). In Figure 3B and Figure 3D, the spawning events are associated only with the zero declinations (rectangles). Furthermore, spawning events at Kochi are associated only with $\mathrm{E}(\mathrm{S}>\mathrm{N})$ while those at Lizard Island are associated only with $\mathrm{E}(\mathrm{N}>\mathrm{S})$ i.e. the zero declination in which the Moon is moving towards the spawning hemisphere.

Another possible influence of the zero declination is evident in that the nearest full moon is consistently shared in each year (2000-2016) with the zero declination dates prior to the mass spawning in both hemispheres (Table S3 and S4: http://doi.org/10.4225/28/59658419a8ee9). For example, the Kochi E(S>N) zero 
declinations on 4 April and 1 May 2000 share the nearest full moon on 18 April 2000. Similarly, the Lizard Island E(N>S) zero declinations on 28 September and 25 October 2000 share the nearest full moon on 13 October 2000.

\section{Atmospheric pressure}

The zero declinations are associated with variations in atmospheric pressure. In all cases, spawning months coincide with low or increasing atmospheric pressure (Figure 4). This includes the months of mass spawning at Kochi and Lizard Island, and the subsequent months after the mass spawning at Lizard Island. All the points in the graphs are zero declinations taken from all lunar cycles in 2000-2016, mapped against days before or after the full moon. On the ordinates are plotted the values of the atmospheric pressures at the times of the zero declinations. The atmospheric pressure is high before the full moon and low after the full moon, coinciding with the third lunar quarter and the Moon moving towards the spawning locations of Kochi $((\mathrm{E}(\mathrm{S}>\mathrm{N})$, Figure $4 \mathrm{~A}$ and Lizard Island ((E(N>S), Figure 4B). The patterns of atmospheric pressure are similar for the locations in both hemispheres, with the spawning events recorded in each hemisphere coinciding with low atmospheric pressures.

\section{Wind speed}

Mass spawning at both Kochi and Lizard Island occurred at a range of wind speeds, with no indication that a particular intensity of this factor is important (Figures S1A and S1B: http://doi.org/10.4225/28/59658419a8ee9). 


\section{Discussion}

\section{'Coincidence of Factors' model}

Our main aim in this paper was to propose a new perspective on factors that may contribute to understanding of the timing of mass spawning in corals. We propose that a close association between two lunar events, the third lunar quarter and the zero declination, may influence this timing. The effect of the declination rhythm on the timing of spawning in corals has not previously been investigated. In support of the Coincidence of Factors model we list the following evidence:

(1) Graphing the interval between the third lunar quarter and the zero declination (LQ - ZD) throughout the whole year, shows that the spawning times at Kochi and Lizard Island occur very close to times when (LQ - ZD) values are short, up to 4 days (Figure 3A and 3C).

(2) If, instead of using the zero declination, we use another declination, e.g. a latitude at either extreme declination or a non-spawning phase, spawning does not occur at any time in those conjunctions (Figure 3B and 3D).

(3) Even if the third lunar quarter and the zero declination are used, spawning will not occur if the incorrect zero declination is used, e.g. $\mathrm{E}(\mathrm{S}>\mathrm{N})$ in the southern hemisphere (Figure 3B and 3D).

We also assessed whether the perigee and apogee could be useful lunar rhythms for influencing timing of coral mass-spawning events. The rhythm of these apsides also has a period of 27.32 days. However, this was ineffective. This may be because the gravitational pattern in the apsides is different to that in the declinations (Kvale et al. 1995). A critical feature of our method appears to be the use of two factors with different rhythms. A combination of two lunar factors with different rhythms may also 
influencing spawning events in non-coral marine invertebrates, perhaps in conjunction with other environmental rhythms.

Our calculations show that a feature in a lunar synodic rhythm (i.e. the third lunar quarter) will coincide with a feature in a lunar non-synodic rhythm (i.e. the lunar zero declination) usually once, and occasionally twice in a year in consecutive months. Similarly, mass spawning can also occur twice in a year, when coral populations spawn in consecutive months (i.e. split spawning) (Willis et al. 1985; Baird et al. 2009) as seen in the Kochi data (Nozawa 2012). Baird et al. (2009) describe how split spawning years are inserted every 2-3 years to realign spawning rhythms, suggesting this as a mechanism for maintaining annual mass-spawning periods within relatively constrained environmental limits, and confining seasonality of spawning to late spring or early summer months.

We show in Figure 1 and in the video in the Supplemental Data (http://doi.org/10.4225/28/59658419a8ee9) that, just once in a year, the lunar quarter and the zero declination occur close together in two successive months. The perioddifferences will vary from year to year, e.g. in the first year we have 0.8 day before the quarter and 1.41 days after the quarter, while in the following year we have 1.60 days before and 0.61 days after the quarter, and in the third year 0.19 day before and 2.02 days after the quarter, and so on. So, not every coincidence will trigger a split-spawning and may depend on the period-difference value. Nevertheless, it would seem that any realignment of spawning rhythms could be automatically provided for by the coincidence of factors. Further analyses, e.g. the relevance of the shared full moon dates for the zero declinations prior to the mass spawning events, are required to assess the relationship between the timing of split spawning and lunar rhythms. 
Willis et al. (1985) concluded that split spawning events may be a response to endogenous rhythms interacting with exogenous factors to realign the number of synodic months separating mass-spawning periods to keep them within acceptable environmental limits. Endogenous factors such as hormonal control of the timing of gonad maturation, as seen in the peak of endogenous steroids in corals prior to mass spawning (Twan et al. 2003, 2006), or the influence of molecular signaling pathways and melanopsin/neuropeptides on the timing of gamete release (Kaniewska et al. 2015) are also important and may be influenced by an endogenous circalunar timer.

Differences in the expression of biological clock genes in corals with respect to lunar cycles have also been detected (Brady et al. 2016; Oldach et al. 2017).

Guest et al. (2005) document evidence of multi-species mass spawning events in equatorial Singapore (1-2 degrees north of the equator) and suggest that environmental factors such as rise in sea temperature, seasonal changes in solar irradiance or changes in the time of sunrise and sunset may be regulating factors. Given that zero declinations are defined by the passing of the Moon over the equator as it moves between each hemisphere, further evidence supporting the Coincidence of Factors model may be obtained by examining the timing of spawning at equatorial locations.

\section{Influence of atmospheric pressure on timing of spawning}

How does the conjunction of the third lunar quarter and zero declination trigger spawning and what environmental factors may be involved? It is well known that the synodic rhythm of the Moon exerts a marked gravitational effect on the Earth (Kvale et al. 1995), greatest during the spring tides at the times of the new and full moons, and least during the neap tides during the lunar quarters. Given that the spawning times in this study correspond with the third lunar quarter and therefore neap tides, we suggest that gravity may have a positive effect on coral spawning because the reduced currents 
during neap tides would be expected to decrease gamete dispersal and thereby increase the chance of fertilisation (van Woesik 2010).

It has recently been discovered that there is a lunar rhythm in the atmospheric pressure. The period of this rhythm is about 27.3 days (Li et al. 2011), similar to that of the declination rhythm of 27.32 days. Li et al. (2011) claim that the gravitational effect of the Moon is greatest at the zero declination i.e. the point at which the Moon crosses the equator as it moves from the northern to the southern hemisphere, and as it moves from the southern to the northern hemisphere. Thus, there appears to be a conflict between the two factors, one decreasing atmospheric pressure i.e. the lunar quarter, and the other increasing it i.e. the zero declination.

For both Kochi and Lizard Island, spawning occurred at or just after the low atmospheric pressure, around the time of the third lunar quarter in both hemispheres. This suggests a possible relationship between spawning and atmospheric pressure and perhaps other associated phenomena (e.g. storms). The apparent conflict between the decrease in atmospheric pressure due to lunar quarters and the elevation in atmospheric pressure associated with zero declinations claimed by Li et al. (2011), was debated by Burke et al. (2013) who found there were increases in human visual acuity at lower atmospheric pressures followed about 1.4 days later by decreases in visual acuity at a higher atmospheric pressure. A similar phenomenon may apply to the coral spawning data. The most likely interpretation is that the main trigger for spawning is the third lunar quarter, assisted by the zero declination irrespective of its timing. The zero declination might also influence spawning through its association with atmospheric pressure. As endogenous factors such as hormones and molecular signalling pathways are important for gonad maturation and spawning (Twan et al. 2003, 2006; Kaniewska 
et al. 2015, Brady et al. 2016; Oldach et al. 2017), the possibility that the zero declination may also influence these biological factors warrants investigation.

With respect to our two case studies, low wind speed, was not evident as a possible influence for the timing of spawning. This is in contrast to Van Woesik (2010) who suggested that calm weather as indicated by low wind speed may be an ultimate cue that triggers coral mass spawning, as a mechanism to promote local retention of gametes and leading to increased fertilisation. In addition Keith et al. (2016) also suggest that moderate wind may be an important environmental cue, along with a rapid increase in sea surface temperature. Following from these observations, further assessment of wind speed, together with atmospheric pressure during coral mass spawning, is warranted.

In this study, we present a possible explanation for the timing of coral mass spawning within a spawning season, focussing on why mass spawning is limited to only a few consecutive days in the year. We suggest that the mechanisms operating on this timing may be influenced by the Coincidence of Factors model with the two factors being a synodic rhythm, the third lunar quarter, and a non-synodic rhythm, the lunar zero declination. Atmospheric pressure could also influence the nights that corals will spawn. We hope that the Coincidence of Factors model will be further explored with detailed data sets on the timing of annual mass spawning in corals and other marine invertebrates to improve our ability to predict these events.

\section{Acknowledgements}

We thank Dr Phil Wilkinson for advice in selecting astronomical sources, HackyHour @ James Cook University staff for assistance with accessing and preparing data files for analysis, Professor John Robinson for statistical advice, and Dr Dane King and Clive 
Jeffery for technical assistance with illustrations. We thank the reviewers for insightful comments that assisted with the revision of the manuscript. 


\section{References}

Alino PM, Coll JC. 1989. Observations of the synchronized mass spawning and post settlement activity of octocorals on the Great Barrier Reef, Australia: biological aspects. Bulletin of Marine Science, 45: 697-707.

Babcock R, Bull G, Harrison P, Heyward A, Oliver J, Wallace C, Willis B. 1986. Synchronous spawnings of 105 scleractinian coral species on the Great Barrier Reef. Marine Biology, 90: 379-394. 10.1007/BF00428562

Babcock R, Mundy C, Keesing J, Oliver J. 1992. Predictable and unpredictable spawning events: in situ behavioural data from free-spawning coral reef invertebrates. Invertebrate Reproduction and Development, 22: 213-227. 10.1080/07924259.1992.9672274

Babcock R, Wills B, Simpson C. 1994. Mass spawning of corals on a high latitude coral reef. Coral Reefs, 13: 161-169. 10.100/BF00301193

Baird, AH, Guest JR, Willis BL. 2009. Systematic and biogeographical patterns in the reproductive biology of scleractinian corals. Annual Review of Ecology, Evolution, and Systematics, 40: 551-571. 10.1146/annurev.ecolsys.110308.120220

Balogh R, Wolfe K, Byrne M. 2018. Gonad development and spawning of the vulnerable commercial sea cucumber, Stichopus hermanni, in the southern Great Barrier Reef. Journal of the Marine Biological Association of the United Kingdom, (accepted 16 January 2018.

Brady AK, Willis BL, Harder LD, Vize PD. 2016. Lunar phase modulates circadian gene expression cycles in the broadcast spawning coral Acropora millepora. Biological Bulletin, 230: 130-142. 10.1086/BBLv230n2p130

Burke W, Davey DF, Leung J. 2013. Lunar influence on human visual acuity: effects 
during the lunar quarters. Biological Rhythm Research, 44: 609-620.

$10.1080 / 09291016.2012 .721688$

Caspers H. 1984. Spawning periodicity and habitat of the palolo worm Eunice viridis

(Polychaeta: Eunicidae) in the Samoan Islands. Marine Biology, 79: 229-236.

10.1007/BF00393254

Dan K, Kubota H. 1960. Data on the spawning of Comanthus japonica between 1937 and 1955. Embryologia, 5: 21-37. 10.1111/j.1440-169X.1960.tb00264.x

Espenak, F. 2012. NASA eclipse website: eclipses and the moon's orbit. [WWW document]. URL http://eclipse.gsfc.nasa.gov/SEhelp/moonorbit.html

Friedlaender B. 1898. Notes on the palolo. The Journal of the Polynesian Society, 7: 4446. $10.2307 / 20701490$

Guest JR, Baird AH, Goh BPL, Chou LM. 2005. Seasonal reproduction in equatorial reef corals. Invertebrate Reproduction and Development, 48:207-218. $10.1080 / 07924259.2005 .9652186$

HackyHour @ James Cook University. 2017. Collaborative DOI description document. Open Science Framework. 10.17605/OSF.IO/Z3XTS

Harrison PL, Babcock RC, Bull GD, Oliver JK, Wallace CC, Willis BL. 1984. Mass spawning in tropical reef corals, Science. 223: 1186-1189.

10.1126/science.223.4641.1186

Harrison P. 2011. Sexual reproduction of scleractinian corals. In: Dubinsky, Z.

Stambler, N, editors. Coral reefs: an ecosystem in transition. Springer, Netherlands, pp. 59-85. 
Hayashibara T, Shimoike K, Kimura T, Hosaka S, Heyward A, Harrison P, Kudo K, Omori M. 1993. Patterns of coral spawning at Akajima Island, Okinawa, Japan. Marine Ecology Progress Series, 101: 253-262.

Himmelman JH, Dumont CP, Gaymer CF, Vallières C, Drolet D. 2008. Spawning synchrony and aggregative behaviour of cold-water echinoderms during multi-species mass spawnings. Marine Ecology Progress Series, 361: 161-168. 10.3354/meps07415 Kaniewska P, Alon S, Karako-Lampert S, Hoegh-Guldberg O, Levy O. 2015. Signaling cascades and the importance of moonlight in coral broadcast mass spawning. eLife, 4.e09991.10.7554/eLife.09991

Keith SA, Maynard JA, Edwards AJ, Guest JR, Bauman AG, van Hooidonk R, Heron SF, Berumen ML, Bouwmeester J, Piromvaragorn S, Rahbek C, Baird AH. 2016. Coral mass spawning predicted by rapid seasonal rise in ocean temperature. Proceedings Royal Society B, 283: 20160011.10.1098/rspb.2016.0011

Korringa P. 1947. Relations between the moon and periodicity in the breeding of marine animals. Ecological Monographs, 17: 347-381. 10.2307/1948665

Korringa P. 1957. Lunar periodicity. Memoir of the Geological Society of America, 67: 917-933. 10.1130/MEM67V1-p917

Kvale EP, Cutright J, Bilodeau D, Archer A, Johnson HR, Pickett B. 1995. Analysis of modern tides and implications for ancient tidalites. Continental Shelf Research, 15: 1921-1943. 10.1016/0278-4343(95)00001-H

Levitan DR, Fukami H, Jara J, Kline D, McGovern TM, McGhee KE, Swanson CA, Knowlton N, Bonhomme F. 2004. Mechanisms of reproductive isolation among 
sympatric broadcast-spawning corals of the Montastraea annularis species complex. Evolution, 58: 308-323. 10.1111/j.0014-3820.2004.tb01647.x

Li GQ, Zong HF, Zhang QY. 2011. 27.3-day and average 13.6-day periodic oscillations in the Earth's rotation rate and atmospheric pressure fields due to celestial gravitation forcing. Advances in Atmospheric Sciences, 28: 45-58. 10.1007/s00376-010-0011-6

Mercier A, Hamel J-F. 2010. Synchronized breeding events in sympatric marine invertebrates: role of behavior and fine temporal windows in maintaining reproductive isolation. Behavioral Ecology and Sociobiology, 64: 1749-1765. 10.2307/40962419

Mercier A, Hamel J-F. 2014. Lunar periods in the annual reproductive cycles of marine invertebrates from cold subtitle and deep-sea environments. In: Numata, H, Helm, B, editors. Annual, Lunar, and Tidal Clocks. Springer, Tokyo, pp. 99-120.

Mezaki T, Hayashi T, Iwase F, Nakachi S, Nozawa Y, Miyamoto M, Tominaga M. 2007. Spawning patterns of high latitude scleractinian corals from 2002 to 2006 at Nishidomari, Otsuki, Kochi, Japan (in Japanese with English abstract). Kuroshio biosphere: Bulletin of the Biological Institute on Kuroshio, 3: 33-47.

Nozawa Y. 2012. Annual variation in the timing of coral spawning in a high-latitude environment: influence of temperature. Biological Bulletin, 222: 192-202. 10.1086/BBLv222n3p192

Okuda S. 1939. Annelida Polychaeta in Onagawa Bay and its vicinity II. Polychaeta errantia with some addenda of Polychaeta sedentaria. Reports of the Tohoku Imperial University, 14: 219-244.

Oldach MJ, Workentine M, Matz MV, Fan T-Y, Vize PD. 2017. Transcriptome 
dynamics over a lunar month in a broadcast spawning acroporid coral. Molecular Ecology, 26: 2514-2526. 10.1111/mec.14043

Sweeney AM, Boch CA, Johnsen S, Morse DE. 2011. Twilight spectral dynamics and the coral reef invertebrate spawning response. Journal of Experimental Biology, 214: 770-777. 10.1242/jeb.043406

Tessmar-Raible K, Raible F, Arboleda E. 2011. Another place, another timer: marine species and the rhythms of life. Bioessays, 33: 165-172. 10.1002/bies.201000096

Twan W-H, Hwang J-S, Chang C-F. 2003. Sex steroids in scleractinian coral, Euphyllia ancora: implication in mass spawning. Biology of Reproduction, 68: 2255-2260. 10.1095/biolreprod.102.012450

Twan W-H, Hwang J-S, Lee Y-H, Wu H-F, Tung Y-H, Chang C-F. 2006. Hormones and reproduction in scleractinian corals. Comparative Biochemistry and Physiology Part A: Molecular and Integrative Physiology, 144: 247-253. 10.1016/j.cbpa.2006.01.011 van Woesik R. 2010. Calm before the spawn: global coral spawning patterns are explained by regional wind fields. Proceedings Royal Society B, 277: 715-722. $10.2307 / 40506175$

Willis B, Babcock R, Harrison P, Oliver J, Wallace C. 1985. Patterns in the mass spawning of corals on the Great Barrier Reef from 1981 to 1984. Proceedings Fifth International Coral Reef Congress, Tahiti, 4: 343-348.

Wolstenholme J. 2004. Temporal reproductive isolation and gametic compatibility are evolutionary mechanisms in the Acropora humilis species group (Cnidaria; Scleractinia). Marine Biology, 144: 567-582.10.1007/s00227-003-1209-2 


\section{Figure legends}

Figure 1. Diagram to illustrate the concept of the 'Coincidence of Factors' model. The large circles represent equatorial cross-sections of the Earth. The small black circles are symbols for the third lunar quarters (synodic: LQ: period 29.53 days). The small black rectangles are symbols for the lunar zero declinations (non-synodic: ZD: period 27.32 days). The symbols orbit the Earth in a clockwise direction. In diagram (A) the symbols are shown in a simultaneous position as a starting point for simplicity (Note: exact simultaneous occurrence is extremely rare). After one lunar cycle (B) the synodic symbol is back in its original position (29.53 days) but the ZD symbol is 2.21 days ahead (29.53 - 27.32). After 3 lunar cycles (C) the ZD symbol is now 6.63 (3x2.21) days ahead. Diagrams (D) and (E) show the ZD symbols after 6 and 9 lunar cycles. At 13 lunar cycles (F) the ZD symbol is now 28.73 (2.21 x 13) days ahead. It is now very close to the synodic symbol $(29.53-28.73=0.80)$ day, and this is assumed to be close enough to satisfy the 'Coincidence of Factors' requirement. After 14 lunar cycles the ZD symbol is now 30.94 (2.21 x 14) days ahead (open rectangle in (F)), but this is equivalent to being only 1.41 (30.94 - 29.53) days away from the synodic LQ symbol and therefore is assumed to also satisfy the 'Coincidence of Factors' requirement. See also the video in the Supplementary Data (http://doi.org/10.4225/28/59658419a8ee9).

Figure 2. Number of coral colonies recorded spawning relative to the third lunar quarter (Day 0). (A) Kochi for each year (2006-2009). Black indicates spawning in July, white indicates spawning in August. Note that only the 'large' spawning events, as recorded in Nozawa (2012), are included. (B) Lizard Island for each year (1999-2001). Black: indicates spawning in November, white: indicates spawning in December, diagonals to the right: spawning in January, diagonals to the left: spawning in February. Data are summarised in Tables S1 (Kochi) and S2 (Lizard Island) 
(http://doi.org/10.4225/28/59658419a8ee9), including the species identification of each colony.

Figure 3. Annual plots of the third lunar quarter (LQ) and zero declination (ZD) gaps for one spawning year for each location: 2007 for Kochi (A, B) and 2000 for Lizard Island (C, D), using four different declinations: when the Moon is at the extreme north $\left(\sim 28^{\circ}\right)$ (open triangle) and extreme south $\left(\sim 28^{\circ}\right)$ (open diamond), and crossing the equator (solid and open circles). Solid circles are used for the declination when the Moon is moving towards the spawning hemisphere: $(\mathrm{E}(\mathrm{S}>\mathrm{N})$ for Kochi and $(\mathrm{E}(\mathrm{N}>\mathrm{S})$ for Lizard Island; open circles as the Moon is moving away from the spawning hemisphere $(\mathrm{E}(\mathrm{N}>\mathrm{S})$ for Kochi and $(\mathrm{E}(\mathrm{S}>\mathrm{N})$ for Lizard Island. The Coincidence of Factors model proposes that the close occurrence of these two lunar factors (LQ and ZD) exerts an important influence on the timing of the annual spawning of corals. The abscissa shows the calendar days, day1 being 1 January, day 365 being 31 December (day 366 in years 2000 and day 365 in 2007). In all graphs the abscissa extends beyond a single calendar year but none of these extensions include a spawning. (A) Kochi and (C) Lizard Island: the LQ-ZD difference throughout a calendar year is superimposed with the dates of spawning (vertical dashed lines). (B) Kochi and (D) Lizard Island: the rectangles mark the temporal limits of the spawning times in all years, with the length of the rectangles indicating the duration of the spawning season and the height of the rectangles indicating the size of the LQ-ZD gap. Note that the spawning dates are restricted to the zero declinations, $\mathrm{E}(\mathrm{S}>\mathrm{N})$ for Kochi (3B) and $\mathrm{E}(\mathrm{N}>\mathrm{S})$ for Lizard Island (3D).

Figure 4. Relationship between spawning, lunar zero declination and atmospheric pressure for (A) Kochi and (B) Agincourt Reef as a proxy for Lizard Island. In each 
graph the individual points plot, on a synodic time scale (Day 0: full moon), the mean daily atmospheric pressure on each day of a zero declination throughout all lunar cycles from 2000 to 2016. In Figure 4(A), the zero declination is $E(S>N)$, i.e. the Moon has come from the southern hemisphere while in Figure 4 (B) the zero declination is $\mathrm{E}(\mathrm{N}>\mathrm{S})$, i.e. the Moon has come from the northern hemisphere. The atmospheric pressure for each lunar zero declination is indicated by crosses in non-spawning months, filled circles in mass spawning months and open circles in subsequent spawning months at Lizard Island. Spawning months for Kochi are based on records from Nozawa (2012) and Mezaki et al. (2007). Spawning months for Lizard Island are based on records from Wolstenholme (2004). As mentioned in the Results, mass spawning months are close to the third lunar quarters (7.5 days after the full moon). Note that the atmospheric pressure pattern is similar in all spawning sites, with the spawning events occurring close to or just after the lowest atmospheric pressure. 


\section{Tables}

Table 1: Spawning events and Zero Declination and Lunar Quarter gaps for Kochi, Japan and Lizard Island, Australia

Table 1A: Kochi

\begin{tabular}{lllllll}
\hline Year & $\begin{array}{l}\text { Spawning } \\
\text { day } \\
\text { (calendar } \\
\text { day) }\end{array}$ & $\begin{array}{l}\text { Number of } \\
\text { colonies }\end{array}$ & $\begin{array}{l}\text { Nearest } \\
\text { LQ day to } \\
\text { spawning } \\
\text { day }\end{array}$ & $\begin{array}{l}\text { Nearest (LQ } \\
- \text { ZD) to } \\
\text { spawning } \\
\text { day }\end{array}$ & $\begin{array}{l}\text { Exact } \\
\text { (LQ - ZD) } \\
\text { to spawning } \\
\text { day }\end{array}$ & $\begin{array}{l}\text { ZD } \\
\text { (calendar } \\
\text { day) }\end{array}$ \\
\hline 2006 & 198 & 37 & 199 & -3 & -2.9 & 196 \\
2006 & 228 & 10 & 228 & -4 & -4.0 & 224 \\
2007 & 190 & 17 & 189 & -3 & -2.8 & 187 \\
2007 & 217 & 18 & 218 & -4 & -4.3 & 214 \\
2008 & 206 & 27 & 208 & -4 & -3.8 & 204 \\
2009 & 198 & 30 & 196 & -3 & -3.4 & 193 \\
\hline
\end{tabular}

Table 1B: Lizard Island

\begin{tabular}{lllllll}
\hline Year & $\begin{array}{l}\text { Spawning } \\
\text { day } \\
\text { (calendar } \\
\text { day) }\end{array}$ & $\begin{array}{l}\text { Number of } \\
\text { colonies }\end{array}$ & $\begin{array}{l}\text { Nearest } \\
\text { LQ day to } \\
\text { spawning } \\
\text { day }\end{array}$ & $\begin{array}{l}\text { Nearest } \\
\text { (LQ - ZD) } \\
\text { to } \\
\text { spawning } \\
\text { day }\end{array}$ & $\begin{array}{l}\text { Exact } \\
\text { (LQ - ZD) to } \\
\text { spawning day }\end{array}$ & $\begin{array}{l}\text { ZD } \\
\text { (calendar } \\
\text { day) }\end{array}$ \\
\hline 1999 & 334 & 15 & 334 & 1 & 1.32 & 338 \\
2000 & 26 & 3 & 28 & 2 & 1.75 & 26 \\
2000 & 324 & 4 & 324 & 0 & 0.23 & 326 \\
2000 & 354 & 1 & 351 & -2 & -1.90 & 354 \\
2000 & 323 & 14 & 324 & 1 & 0.98 & 326 \\
2001 & 14 & 4 & 16 & 1 & 0.95 & 15 \\
2001 & 44 & 10 & 42 & 4 & 3.83 & 42 \\
2001 & 340 & 20 & 343 & 4 & 4.33 & 343 \\
\hline
\end{tabular}


Figure 1
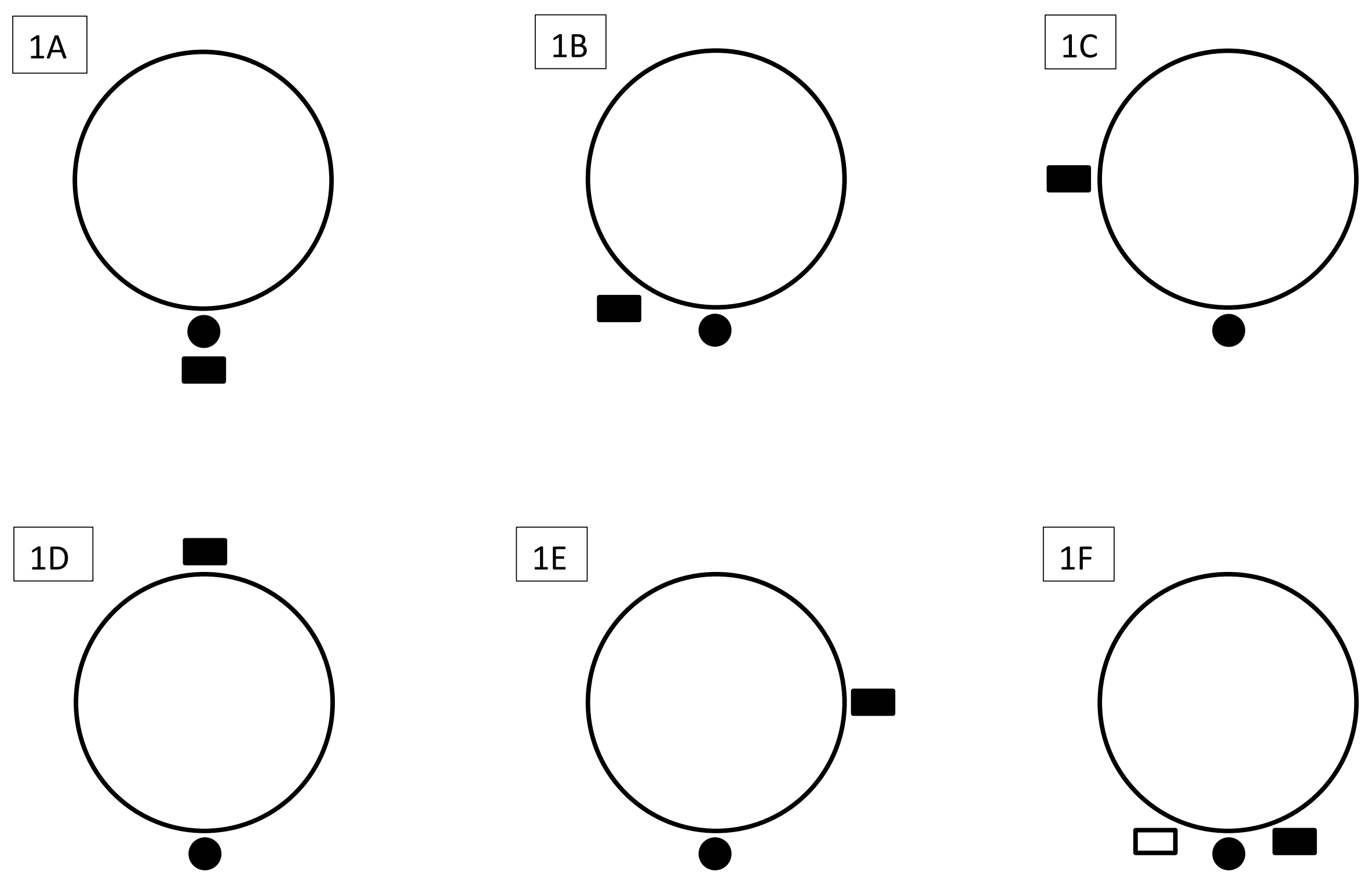
$2 \mathrm{~A}$
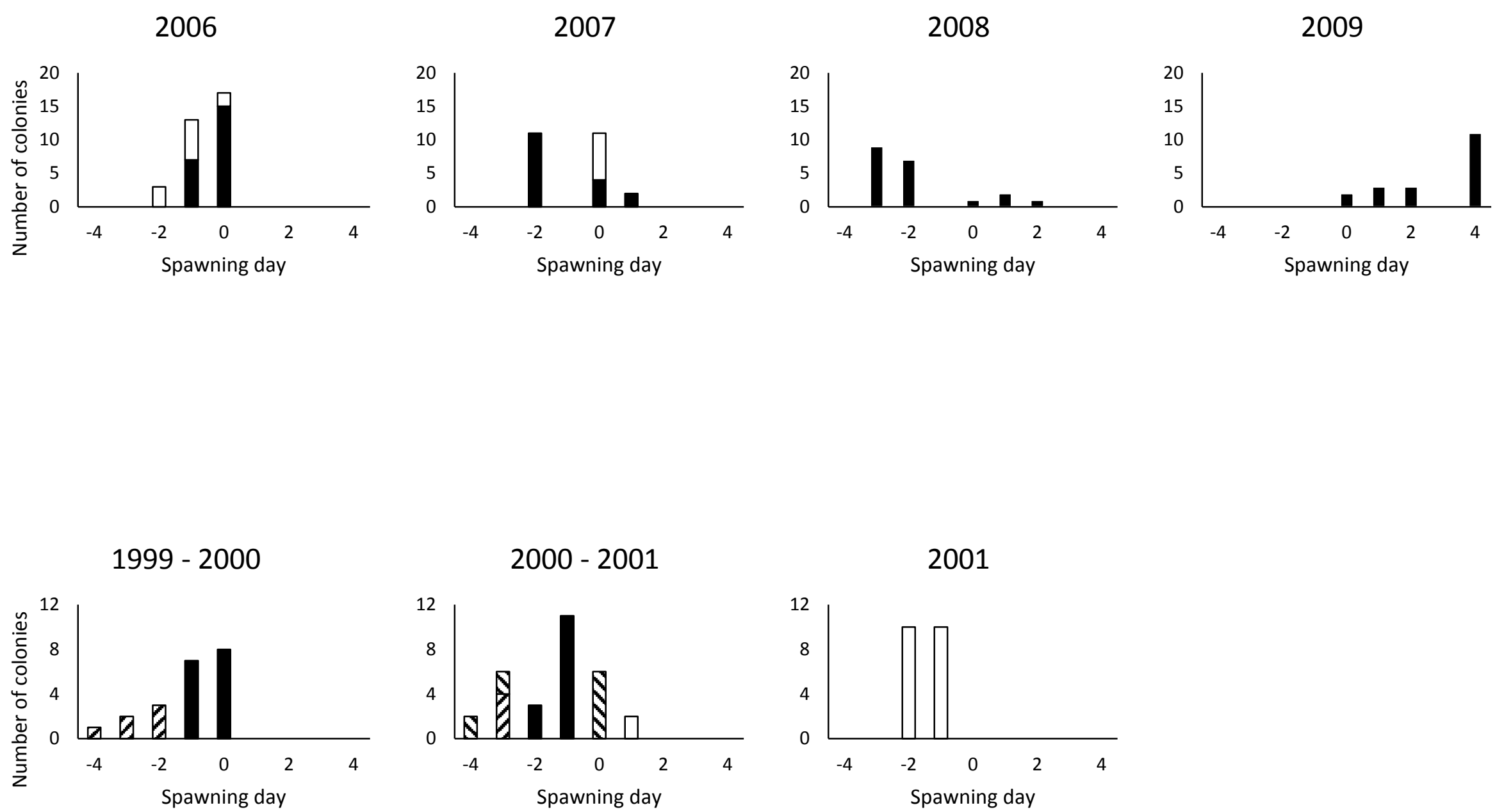


$$
3 A
$$

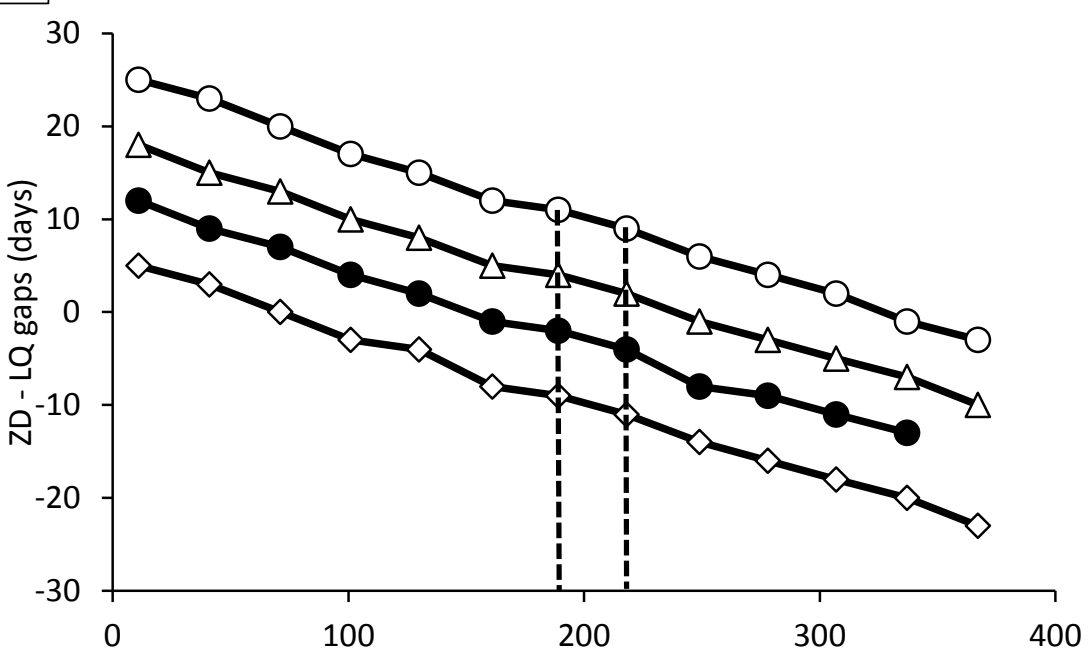

\section{$3 C$}

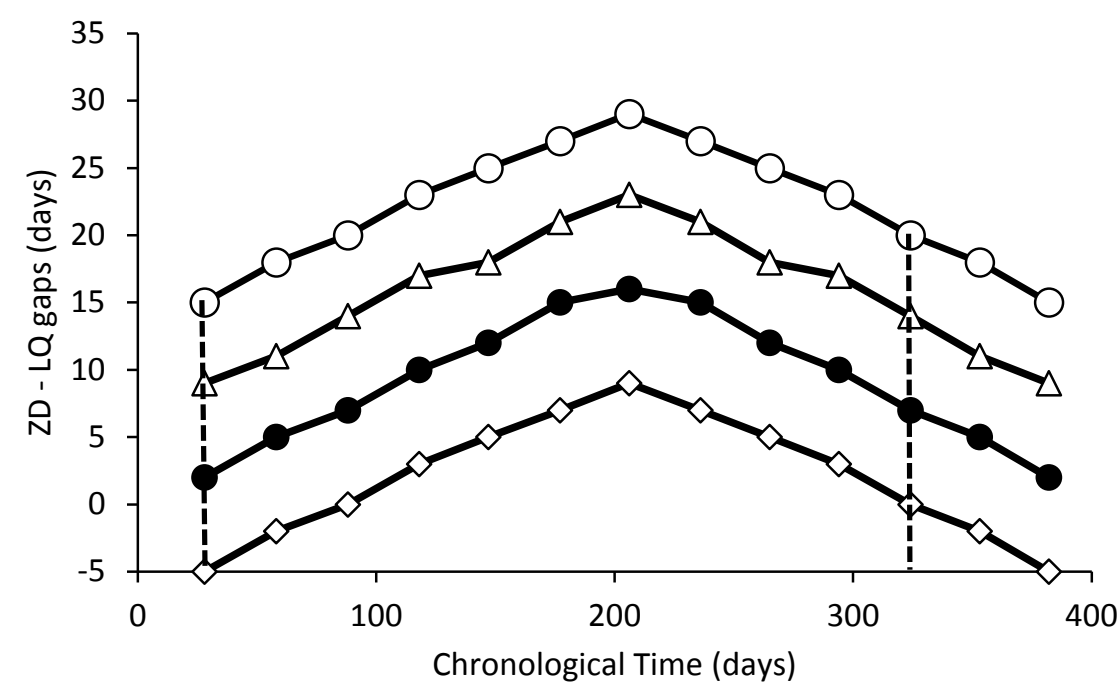

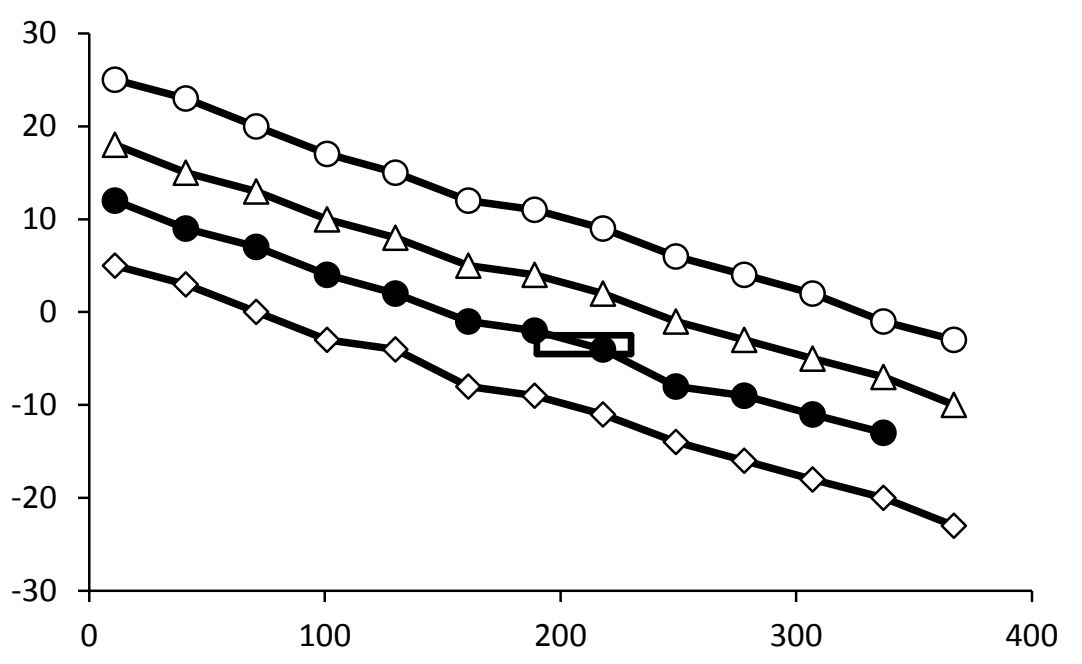

\section{D}

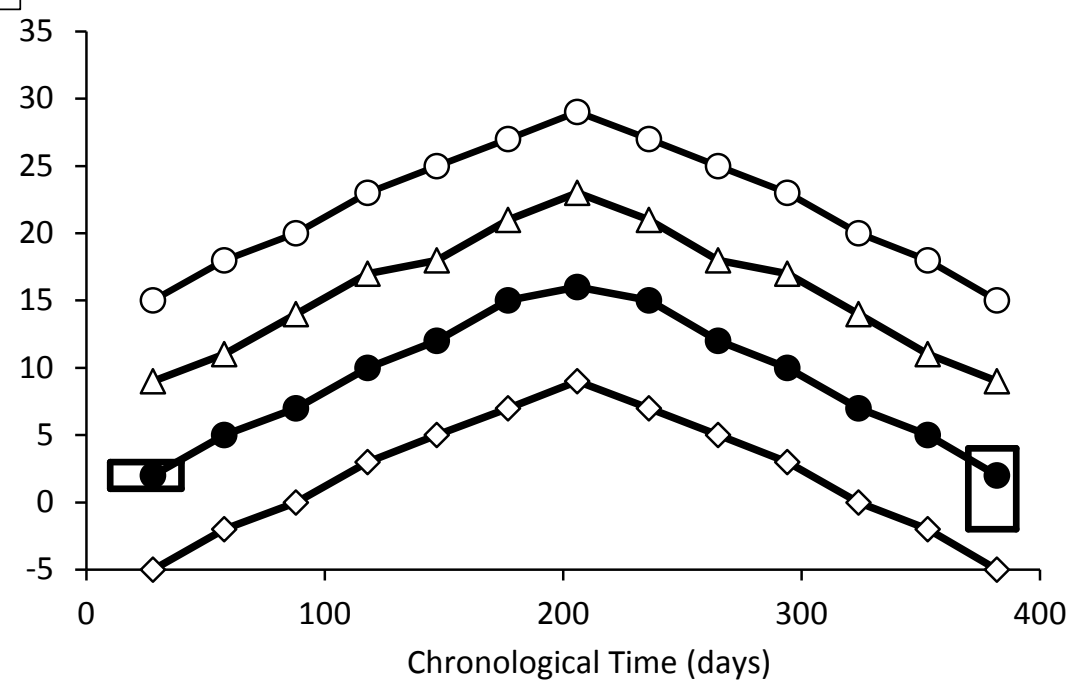



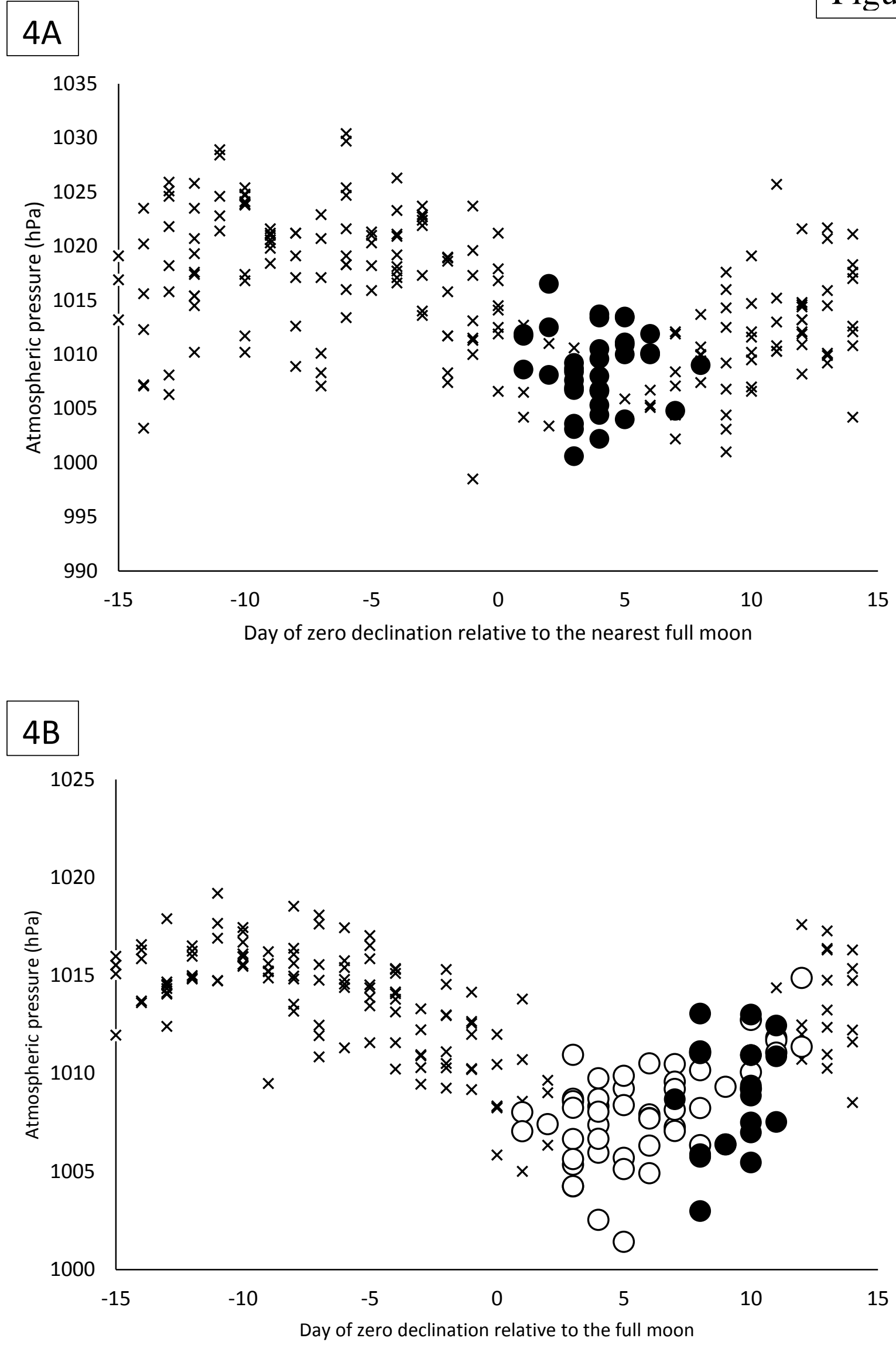